\title{
Analysis of Noise Environment and Noise Reduction Measures in Urban 110kV Indoor Substations
}

\author{
Fengchun Liu ${ }^{1 *}$, Shan Tu${ }^{1}$, Hanbing Yan ${ }^{1}$, Xiufang Chen ${ }^{1}$, Liqiang Guo ${ }^{1}$ \\ ${ }^{1}$ Electric Power Research Institute, Shanxi Provincial Electric Power Corp. Taiyuan, Shanxi Province, 030001, China
}

\begin{abstract}
With the rapid development of society and economy, the public awareness of environmental protection has gradually increased, and urban substation noise has become an environmental issue of concern to residents. In order to study the current status of the noise environment of urban substations, to guide the management of substation noise and the construction of transmission and transformation projects, this article selects $50110 \mathrm{kV}$ indoor substations as objects, and measures their boundary noise. By analyzing the characteristics of the noise source of the substation with excessive noise and various noise reduction measures, the noise reduction methods suitable for urban indoor substations are summarized.
\end{abstract}

\section{Introduction}

With the rapid economic development, electricity consumption is growing rapidly. In order to meet the needs of social development, substations are built near the urban centers, close to the residents' working and living areas. With the improvement of public awareness of environmental protection, the impact of urban substation on life has been paid more and more attention [1-3].

A sample of $50110 \mathrm{kV}$ urban substations in a province was selected for noise testing and analysis, and the main noise sources of the substation were studied. Aiming at the main noise sources, the corresponding noise reduction measures are studied to provide guidance for the noise control of existing substations and the design of new substations.

\section{Substation noise test plan}

This article selects $50110 \mathrm{kV}$ indoor indoor substations as the research object, describes the noise level of the substation by measuring the noise at the boundary of the plant, and uses the NL-52 type sound level meter for on-site measurement. Take readings in the sound level state. Set the time weighting feature of the instrument to "F", select the mode as "Leq", and read the equivalent continuous A sound level for 1 minute. The number of measurements at each point is not less than 3 times, and the interval between each measurement is not more than $5 \mathrm{~min}$. The test layout follows the following principles:

a) Place points evenly around the station boundary. The difference in noise measurement between two adjacent points should not be greater than $3 \mathrm{~dB}$ (A). If it is greater than $3 \mathrm{~dB}(\mathrm{~A})$, the points should be encrypted. b) The measurement point should be close to the direction of sound wave propagation of noisy equipment.

c) The measurement point should be selected at a location $1 \mathrm{~m}$ away from the station boundary, a height of $1.2 \mathrm{~m}$ or more, and a distance of not less than $1 \mathrm{~m}$ from any reflecting surface;

\section{Result analysis}

The distribution of the test results of the plant boundary noise of the 50 substations is shown in Table 1 . According to the requirements of the "Environmental Noise Emission Standards for Industrial Plant Factory Boundaries" (GB12348-2008), the limit of noise at factory boundaries is $60 \mathrm{~dB}$ (A) during the day and $50 \mathrm{~dB}$ (A) at night. From the test results, the daytime plant boundary noise of all substations is lower than $60 \mathrm{~dB}(\mathrm{~A})$, which meets the requirements of national standards. The night noise of 5 substations exceeds $50 \mathrm{~dB}$ (A), which does not meet the requirements of national standards.

Table 1. Substation boundary noise distribution

\begin{tabular}{ccccccc}
\hline $\begin{array}{c}\text { Noise } \\
\text { range } \\
\text { dB(A) }\end{array}$ & 40 & $40-45$ & $45-50$ & $50-55$ & $55-60$ & $\begin{array}{c}60 \\
\text { and } \\
\text { above }\end{array}$ \\
\cline { 2 - 7 } $\begin{array}{c}\text { Daytime } \\
\text { noise } \\
(\text { QTY) }\end{array}$ & 0 & 0 & 13 & 35 & 2 & 0 \\
$\begin{array}{c}\text { Night } \\
\text { noise } \\
\text { (QTY) }\end{array}$ & 7 & 12 & 26 & 3 & 2 & 0 \\
\hline
\end{tabular}

In order to understand the cause of excessive noise in substations, we went into the substation for testing to find the main noise source. The five substations are all indoor substations with single buildings and two main transformers inside. The transformers are separated from

\footnotetext{
*Corresponding author's e-mail: 842341737@qq.com
} 
the outside world by the volume gate. After inspection, there are two main sources of substation internal noise, one is transformer noise, the other is building ventilation facilities. We tested the main transformer of 5 substations and the noise of building ventilation facilities to analyze the reasons for the noise exceeding the limit.

Table 2. Noise of main equipment in substation

\begin{tabular}{lccccc}
\hline $\begin{array}{l}\text { Substation } \\
\text { number }\end{array}$ & 1 & 2 & 3 & 4 & 5 \\
\cline { 2 - 6 } $\begin{array}{l}\text { Transformer } \\
\text { noise }\end{array}$ & 54.2 & 68 & 52.9 & 53.6 & 54.2 \\
$\begin{array}{l}\text { Ventilation } \\
\text { facility noise }\end{array}$ & 63.5 & 57.6 & 62.8 & 65.4 & 67.1 \\
\hline
\end{tabular}

The results show that the ventilation facilities of substations $1,2,4$, and 5 are relatively noisy. In order to analyze the impact of building ventilation facilities on plant boundary noise, we closed the ventilation equipment of 5 substations, measured their boundary noise, and tested The results are shown in the table. We found out that after closing the ventilation facilities, the noise level at the plant boundary of No. 1, 2, 4, and 5 will be within the national standard limit. It can be explained that the noise of the building ventilation facilities is the main cause of excessive noise at the plant boundary of No. 1, 2, 4, and 5 substations.

According to the test results in the table, it can be found that the main transformer of No. 2 substation is relatively noisy. After checking the transformer data, it can be known that the main transformer's commissioning time was 1997, the cooling mode was ONAF, and the operating life had exceeded 20 years. The transformer is poorly maintained (Figure 1). Vibration and cooling fan noise are the main noise sources in this substation, and they are also the main cause of excessive substation noise.

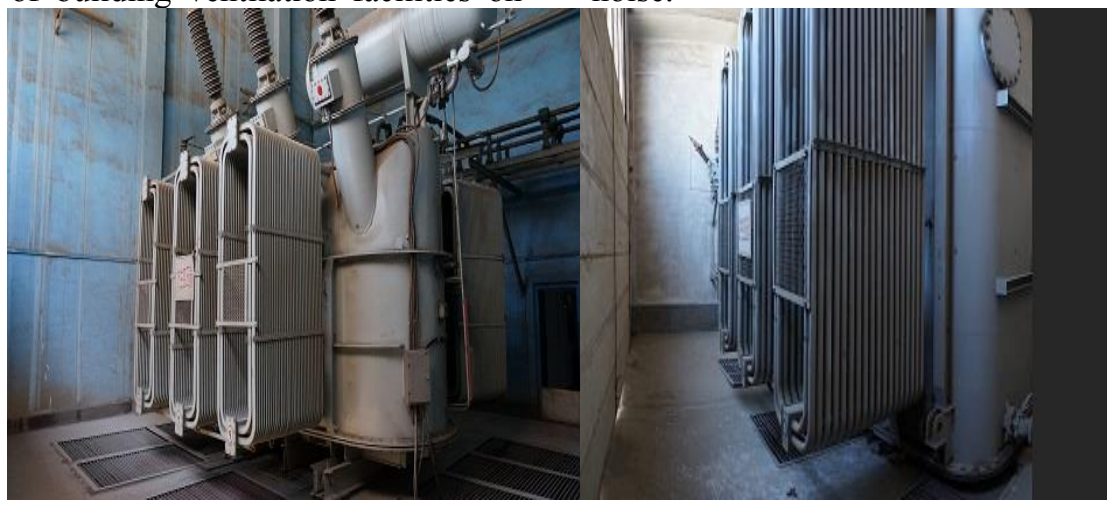

Figure 1. The transformers of No.2substation

The noise of the main transformer is composed of electromagnetic noise and cooling system noise ${ }^{[4-6]}$. The electromagnetic noise is mainly caused by the magnetostriction of the silicon steel sheet, and the frequency of the magnetostrictive change is twice the frequency of the power supply, plus the presence of higher harmonic magnetic flux components, which results in the noise spectrum In addition to the power supply frequency (fundamental frequency), there is an additional frequency that is an integer multiple of the fundamental frequency. The overall performance is mainly low and medium frequency noise. The noise of the cooling system is mainly caused by the fan, and the two kinds of noise are superimposed on each other to form a noise spectrum characteristic mainly composed of low and medium frequency noise and supplemented by high frequency noise ${ }^{[7-9]}$.

The noise caused by the inlet and exhaust air system is often not taken into account in the planning and design of the running substation, and the design is not reasonable and the noise reduction means are not adopted. As a result, the noise of the ventilation system has become an important noise source of the substation. Noise of building ventilation device is mainly from fan noise, including rotating noise and eddy noise, and vibration noise of blinds is also present in part (figure 2). There is no common noise spectrum in the ventilation system, so specific equipment needs to be analyzed.
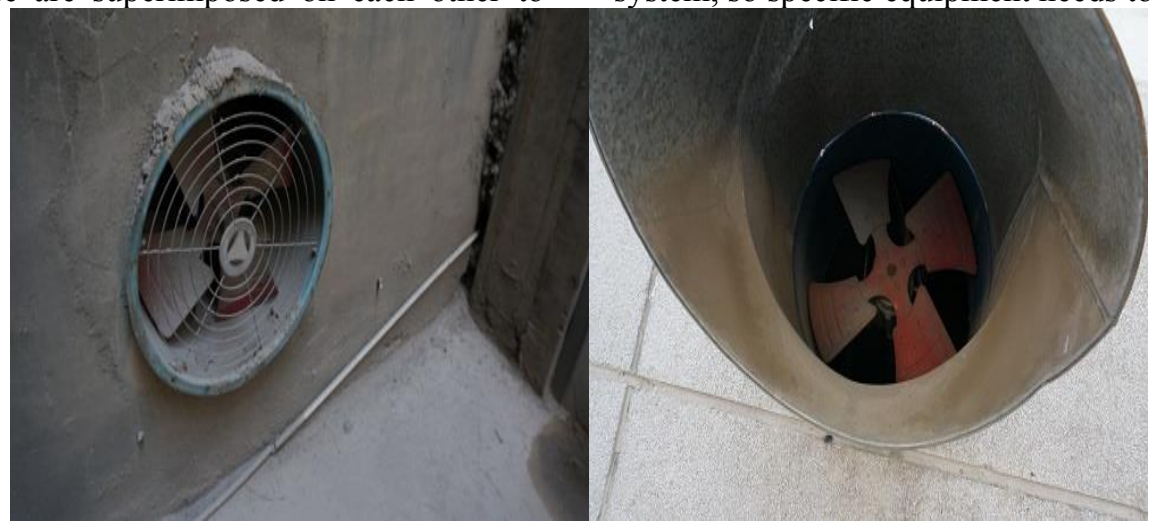


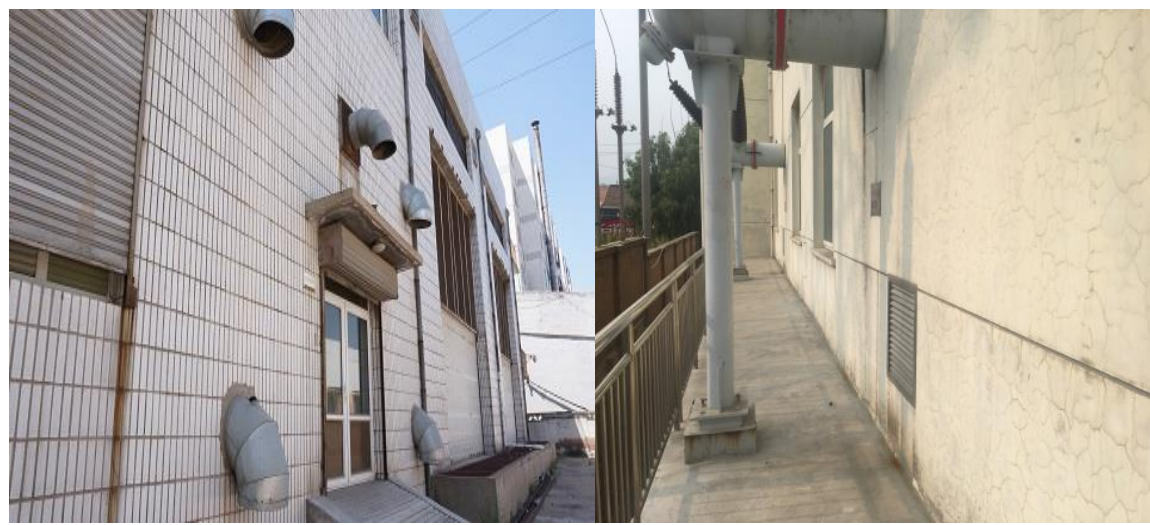

Figure 2. Substation building ventilation facilities

\section{Analysis of noise reduction measures}

\section{1 sound absorption and noise reduction}

When the sound wave encounters various solid materials during the propagation process, part of the sound energy is reflected, part of the sound energy enters the material and is absorbed, and a small part of the sound energy is transmitted to the other side. When the indoor sound source emits noise, in addition to the direct sound from the sound source, people will also hear the reflected sound formed by multiple reflections on various surfaces in the room, which will make people working indoors more exposed. Noise effect. We decorate the ceiling and surrounding walls of the transformer room with a sound absorbing material to increase the attenuation of the main transformer noise and reduce the impact of the transformer noise on the station boundary environment [10].

\subsection{Noise reduction}

Noise reduction is a measure to reduce noise by using airflow ducts with sound-absorbing linings or special structural forms. It has the characteristics of allowing airflow to pass and preventing sound transmission. It is achieved by installing a muffler. Common mufflers include resistive mufflers, resistant mufflers, impedance composite mufflers, micro-perforated plate mufflers, active mufflers, etc. Install mufflers on the ventilation pipes of ventilation and cooling systems such as substation fans to suppress aerodynamic noise levels and reduce the impact of noise on the station boundary environment ${ }^{[11]}$.

\subsection{Sound insulation and noise reduction}

Sound insulation is a technical measure commonly used in noise control engineering. The use of walls, various plates and components as shields or the use of envelope structures to control noise within a certain range makes the propagation of noise in the air blocked and cannot Pass smoothly, so as to achieve the purpose of reducing noise. Install sound insulation devices on the noise propagation channels of main noise source equipment such as main transformers, reactors and ventilation and cooling systems to reduce the environmental noise level at the station boundary ${ }^{[12]}$.

\section{Conclusion}

According to the monitoring and analysis of the noise levels of $50110 \mathrm{kV}$ indoor substations, the main transformers and ventilation facilities are the main sources of noise pollution in urban indoor substations, causing noise levels at some station boundaries to exceed standards. In response to the excessive noise of the station boundary of the substation ${ }^{[13-15]}$, sound-absorbing materials can be installed on the surrounding walls and ceilings, which can effectively reduce the noise generated by the main transformer; choosing a suitable isolation door can also effectively reduce the impact of indoor transformer noise on the station boundary. For building ventilation equipment, the noise mainly comes from fans. The noise reduction methods that can be used include: reducing the generation of noise through structural design; adding a layer of sound-absorbing material on the inner surface of the cabinet; and adding a muffler to the vent.

In addition, substation noise can also be optimized and controlled from the aspects of substation site selection, substation plane layout and layout optimization, and substation equipment selection, so as to realize the harmonious development of human and environment. environment.

\section{References}

1. Liu, M. Cui, Y, B. Chen, C. Shi, B. (2019) Analysis and optimization control of noise pollution for substations. Electric Power Environmental Protection, 35(04): 1-4.

2. Zhou, J, F. Zhou, G, N. Yang, J, C. Lv, J, H. Chen, S, Y. (2011) Research of Typical Noise Control Technology of Substation in Urban Area. Noise and Vibration Control,31(05):173-177.

3. Liu, H. Yin, J, G. Zhang, G, Y. Zhang, Y. Xie, L, K. Ji, Y, F. Liu, G, S. An, S,H.(2018)Analysis of Noise Pollution of $110 \mathrm{kV}$ Outdoor Substation and 
Discussion of Noise Control. Guangdong Electric Power,31(12):12-19.

4. Ji, S, C. Shi, Y, H. Zhang, F. Lu, W, F. (2019) Review on Vibration and Noise of Power Transformer and Its Control Measures. High Voltage Apparatus,55(11):1-17.

5. Wu, X, W. Lu, L. Hu, S. Cao, H. Zeng, H, F. Ou, Y, L. (2019) Statistics and Analysis of Audible Noise Features of $110 \mathrm{kV}$ Transformers. High Voltage Apparatus,55(11):113-117.

6. Gu, X, A. Shen, R, Y. Xu, J, T. (2001Study on the vibration and noise control techniques in large power transformers. Noise and Vibration Control,05:7-11.

7. Zhu, L, H. Yang, Q, X. Yan, R, G. Z hang, X. (2013) Research on Vibration and Noise of Power Transformer Cores Including Magnetostriction Effects. Transactions Of China Electrotechnical Society, 28(04):1-6.

8. Tan, W. Zhang, X, W. (2009) Investigation and Control of Power Transformer Noise. High Voltage Apparatus, 45(02):70-72.

9. Zhu, Y, Y. Ji, S, C. Zhang, F. Liu, Y. Dong, H, K. Cui, Z, G. Wu, J, W. (2015) Vibration Mechanism and Infulence Factors in Power Transformers. Journal Of XI'AN Jiaotong University, 49(06): $115-125$.
10. Liang, D. Zheng, Y. Hong, Q. Chen, F, N. Liang, Z, M. Ding, N. Meng, X, B. (2018) Research on Sound-Absorption Materials and Structure for UHV Substation Noise Reduction. Hebei Electric Power, 37 (05): 1-4.

11. Lu, C, X. Wang, L, M. Chen, S, M. Meng, X, M. $\mathrm{Wu}, \mathrm{H}$. Zhan, G. (2016) Preparation and Sound Absorption Property of Alumina Ceramic Foam for Sound Insulation and Noise Reduction in Transformers. Materials For Mechanical Engineering, 40(09): 22-25.

12. Gao, Z, Y. (2017) Structural design and performance test of muffler for local fan in mine. Coal Engineering, 49 (12): 90-92.

13. Cai, W. She, K. Peng, X, F. Wu, X, W. Yi, M, S. Lu, L. (2019) Noise Control Technology and Its Application in $110 \mathrm{kV}$ Substation. Technology Exchange, 47(09): 120-126.

14. Yi, M, S. Wu, H, L. Cai, W. She, K. Peng, X, F. Wu, $X$, W. (2018) Audible Noise Measurement and Control Technology of a $110 \mathrm{kV}$ Substation in Residential Area. Power Grid Analysis \& Study, 46(11): 83-87.

15. Su, Z, J.Huang, W.(2019) Analysis and treatment of substation noise. Energy Conservation \& Environmental Protection, 06: 61-63. 